\title{
Genetic and Clinicopathologic Characteristics of Papillary Thyroid Carcinoma in the Chinese Population: High BRAF Mutation Allele Frequency, Multiple Driver Gene Mutations, and RET Fusion May Indicate More Advanced TN Stage
}

\author{
Zhihong Wang (D) ${ }^{1, *}$, Peng Tang ${ }^{2} *$, Surong Hua', Junyi Gao', Bin Zhang ${ }^{3}$, Hua Wan $\mathbb{D}^{4}$, Qixi Wu ${ }^{4}$, \\ Jiaxin Zhang ${ }^{5}$, Ge Chen' \\ 'Department of General Surgery, Peking Union Medical College Hospital, Chinese Academy of Medical Sciences, Beijing, People's Republic of China; \\ ${ }^{2}$ Department of Breast and Thyroid Surgery, Southwest Hospital, Third Military Medical University (Army Medical University), Chongqing, People's \\ Republic of China; ${ }^{3}$ Department of General Surgery, Qilu Hospital of Shandong University, Jinan, People's Republic of China; ${ }^{4}$ Research and \\ Development Department, Beijing USCI Medical Laboratory, Beijing, People's Republic of China; ${ }^{5}$ Department of Thyroid and Breast Surgery, \\ Affiliated Hospital of Xuzhou Medical University, Xuzhou, People's Republic of China
}

*These authors contributed equally to this work

Correspondence: Ge Chen, Department of General Surgery, Peking Union Medical College Hospital, Chinese Academy of Medical Sciences, No. I Shuai Fu Yuan Hu Tong, Beijing, 100730, People's Republic of China, Tel +86 I56 II 23 3738, Fax +86 I56 69I52600,

Email chenge@pumch.cn; Jiaxin Zhang, Department of Thyroid and Breast Surgery, Affiliated Hospital of Xuzhou Medical University, No. 99, Huaihai West Road, Xuzhou, 22 1004, People's Republic of China, Tel +86 I80 5226 8693, Fax +86 I8085802306, Email zhangjiaxin I969@I63.com

Purpose: To describe the genetic landscape and clinical characteristics of Chinese patients diagnosed with papillary thyroid cancer (PTC) and to determine which high-risk genetic characteristics suggest a likelihood of lymph node metastasis (LNM) and lateral lymph node metastasis (LLNM).

Patients and Methods: Data from previously untreated patients with PTC collected between May 2018 and December 2020 from 14 hospitals in China were analyzed retrospectively. High-risk pathologic characteristics were defined as T3/T4, N(+), and N1b(+) stages. All patients were tested for 57 genes by second-generation sequencing. The $t$-test, chi-square test, and Fisher's exact test were performed for statistical analysis.

Results: Overall, 395 patients were enrolled in this study. The prevalence of $B R A F$ mutation was $78.53 \%$. BRAF mutant allele frequency $(\mathrm{MAF})>16.93 \%$ was associated with a significantly higher risk of LNM, LLNM, and T3 + T4 stage compared with a lowrisk group, defined by a MAF $<2.54 \%$ (odd ratios [ORs] for each risk=3.38, 3.46, and 8.54, respectively), and an intermediate-risk group, defined by a MAF of $2.54 \%$ to $16.93 \%$ (ORs=2.04, 2.07, and 4.07, respectively). The population with RET fusion had higher T, $\mathrm{N}$, and N1b stages (ORs for each stage $=10.40,7.60$, and 8.77, respectively) compared with a RET-negative population. Similar conclusions about $\mathrm{T}, \mathrm{N}$, and $\mathrm{N} 1 \mathrm{~b}$ stages were observed in relation to multiple driver gene mutations (ORs for each stage $=7.48,2.80$, and 7.04, respectively) compared with population without multiple driver mutations. These genetic characteristics may be suggestive of high clinical risk. However, regardless of genetic profiles, patients younger than age 45 years had greater rates of LNM and LLNM. Conclusion: The main driver gene in this study, BRAF, differs significantly between the United States (79\% vs 51\%) and other countries. The Chinese population in this study that experienced more aggressive tumor biology had a BRAF MAF greater than $16.93 \%$, exhibited RET fusion events, and had multiple driver gene mutations; thus, these traits may be considered high-risk genetic characteristics in PTC that could warrant aggressive treatment in such population.

Keywords: papillary thyroid cancer, lymph node metastasis, high risk clinicopathological characteristics, genotypes

Received: 14 September 2021

Accepted: 2 January 2022

Published: 9 February 2022 


\section{Introduction}

Thyroid cancer is the most common malignant endocrine cancer both worldwide and in China. Its incidence has been gradually increasing in East Asia in recent years. ${ }^{1}$ The age-specific incidence of thyroid cancer in the standard population of China was $8.82 / 100,000$ in $2013,{ }^{2}$ and the mortality rate was $0.52 / 100,000 .{ }^{3}$ In the past few decades, the incidence of papillary thyroid cancer (PTC) - the most common thyroid cancer — has been increasing steadily; now, PTC may account for $>99 \%$ of thyroid cancers in China. ${ }^{4}$ Patients diagnosed with PTC often undergo surgical treatment and postoperative thyroid-stimulating hormone suppressive therapy. The 5-year survival rate after surgery of PTC is $>95 \% .{ }^{5}$ However, contralateral central lymph node metastasis (LNM) is still confirmed in $3.88 \%$ to $30.63 \%$ of the patients who have no LNM detected by preoperative ultrasound or physical examination (cN0 patients) after undergoing total thyroidectomy. ${ }^{6,7}$ Therefore, it is controversial whether prophylactic lymph node prophylaxis in the contralateral central lymph node is required for patients with a preoperative ultrasound diagnosis of cN0.

Genetic mutations could serve as potential predictive factors for LNM or for therapeutic efficacy in PTC. ${ }^{8,9}$ Previous study have revealed that the major mutations in PTC are located in the MAPK pathway (eg, BRAF and RAS mutations, gene fusion events such as RET, NTRK1, and $B R A F$ gene fusions). ${ }^{9}$ Recent research indicates that patients with PTC who have $B R A F$-positive/TERT-positive co-mutations have a significantly high probability and prognosis of distant LNM than do those without detectable gene mutations. ${ }^{10,11}$ Therefore, preoperative genetic testing may help clinicians predict the prognosis of patients and guide clinical decisions.

A large-scale genetic landscape study of PTC in a Chinese population (involving 355 patients) was performed in 2018. ${ }^{12}$ According to this study, the genetic characteristics of the reported population differed significantly from those of patients from the United States and Saudi Arabia, suggesting that the genetic or epigenetic characteristics of PTC may differ across ethnic backgrounds. However, additional studies are needed to confirm this hypothesis. Moreover, this single-center study did not focus on the relationships between genotype and clinical characteristics (eg, LNM). In this retrospective, multicenter study, we reported the clinical and pathologic characteristics of PTC and the genetic profile of patients with primary PTC in a Chinese population. We also explored the association between genetic mutations and clinical or pathologic features to identify high-risk genetic characteristics that may propose a likelihood of LNM and provide data to support individualized diagnosis and treatment strategies.

\section{Patients and Methods}

\section{Patient Enrollment and Screening}

Data from patients diagnosed with thyroid cancer who underwent a 57-gene-panel test from May 2018 to December 2020 were retrospectively collected in this study; 451 patients from a total of 14 tertiary hospitals in seven provinces and cities were included. The following clinical characteristics and pathologic features were collected for each patient: age, sex, personal history, family history, specific surgical procedure, detailed pathologic diagnosis, tumor size, and LNM. Fresh tissues or formalin-fixed, paraffin-embedded (FFPE) samples were collected from each patient during radical thyroidectomy. All samples underwent DNA testing for 57 genes with high mutation frequencies in PTC (Figure S1). After all data were collected, they were classified by T, N, and M stages according to the 2015 American Thyroid Association Management (ATA) guidelines. ${ }^{13}$ The location of the primary site was classified according to the location of the cancer site, as documented by the pathologic diagnosis after the initial thyroidectomy. In this study, locations were classified as unilateral or bilateral, and unilateral locations were classified as left or right. High-risk genetic characteristics were defined as a $\mathrm{T}$ stage $\geq \mathrm{T} 3$, an $\mathrm{N}$ stage $=\mathrm{N} 1$, or an $\mathrm{M}$ stage $=\mathrm{M} 1$.

The target population was previously untreated patients with thyroid cancer and a clear postoperative pathologic diagnosis of PTC according to the criteria defined by the World Health Organization. ${ }^{14}$ During the screening process, 54 patients were excluded: 13 patients underwent non-primary surgery, 17 patients did not have PTC (5 had benign nodules, 8 had medullary carcinomas, 1 had follicular carcinoma, and 4 had undifferentiated carcinomas), and 24 patients had missing pathologic diagnosis data. During next-generation sequencing, 2 patients were excluded from the study because their gene extraction results were ineligible. Overall, 395 patients were eventually included in the data analysis. 
The study protocol was approved by the ethical board of the Chinese Academy of Medical Sciences and Peking Union Medical College Hospital, and all participants gave written informed consent at their local hospitals. This study was conducted in accordance with the Declaration of Helsinki.

\section{Second-Generation Sequencing}

Genomic DNA was isolated from fresh tumor tissues or FFPE using the QIAamp DNA FFPE Tissue Kit (Qiagen) according to the manufacturer's instructions. The concentration of the DNA was determined using the Qubit Fluorometer 3.0 (Life Technologies, USA). A custom-designed, 103-kb panel covering exons and partial introns of cancer driver genes, hereditary cancer-related genes, and therapy-related genes was used in this study. First, 50-100 ng of sheared genomic DNAs was subjected to library construction with an MGIEasy Universal DNA Library Kit (MGI, China), and then the DNA underwent hybrid capture using an xGen Hybridization and Wash Kit (IDT, USA). Libraries' quality and concentration were determined using a LabChip ${ }^{\mathbb{B}}$ GX Touch $^{\mathrm{TM}}$ nucleic acid analyzer (PerkinElmer, USA) and a Qubit Fluorometer 3.0, respectively. Tumor-matched normal samples were sequenced as controls. The qualified libraries were sequenced with $2 \times 100$ bp paired-end reads on an MGISEQ-2000 (MGI, China) platform.

\section{Bioinformatic Analysis}

The sequencing data were mapped to the GRCh37/hg19 human genome using a Burrows-Wheeler Aligner (BWA, v.0.7.12) MEM algorithm. PCR duplicates were marked using Picard tools (v.1.119). Functional annotations of somatic SNVs and InDels were performed using ANNOVAR. Candidate mutations were filtered if (1) the depth was $<200 \times$ for tissues and reads supporting alternative alleles were $<4$; (2) the reads had strand bias; (3) the mutant allele frequency (MAF) was $<0.5 \%$; and (4) the variations were synonymous, with unknown significance and introns.

Gene fusions were detected with an in-house algorithm. Briefly, the relative positions of the aligned paired sequences were compared to find the possible structural variation. The abnormally aligned sequences were segmented and aligned by a more relaxed alignment method to map the sequences to possible positions and determine the final alignment position and direction. To determine the positions of the breakpoint, the candidate final alignment positions were marked, and then the sequences were realigned. Only candidate breakpoints with high alignment quality and with supporting reads that covered the points were retained. Breakpoint correction was performed on the basis of alignment scores, sequencing quality, and number of supported sequences. False positives were filtered by tag reads covering the breakpoints and by paired reads supporting the breakpoints. Requirements to consider a gene fusion valid were as follows: (1) depth $>500 \times$ at the breakpoint; (2) at least eight high-quality unique reads covering the breakpoint; and (3) MAF $\geq 0.5 \%$.

CNVkit was used to identify copy number variants. The minimum threshold of copy number $(\mathrm{CN})$ gain or loss was $\mathrm{CN}$ $>2.75$ or $\mathrm{CN}<1.75$ for hotspot genes, and $\mathrm{CN}>3$ or $\mathrm{CN}<1.5$ for others. Gene rearrangements were assessed using the Integrative Genomics Viewer. Some important/novel variants were verified by Sanger sequencing or ddPCR, if needed.

\section{Statistics Analysis}

Data analysis was conducted with $\mathrm{R}$ version 4.0.3. The $t$-test was applied for comparison of means, and certain proportions in specific circumstances. As for categorical variables, we properly take advantage of chi-square test and Fisher's exact test in comparison between two variables. Cochran-Mantel-Haenszel test and exact conditional test, their counterparts in three variables' situation, were used in aforesaid condition. A p-value of $<0.05$ was considered as significant.

\section{Results}

\section{Overview of Clinicopathologic Characteristics Genetic Profile in Chinese PTC Patients: $B R A F^{V 600 E}$ Mutation is the Majority Mutation in Chinese Population}

Overall, 395 patients from different provinces in China were included in this study. The mean \pm standard deviation age was $44.43 \pm 12.25$ years, and the gender ratio was nearly 3:1 (296 women and 99 men). The tumors were located on the left, right, and both sides in 133, 166, and 96 patients, respectively. Interestingly, PTC presented less often on 

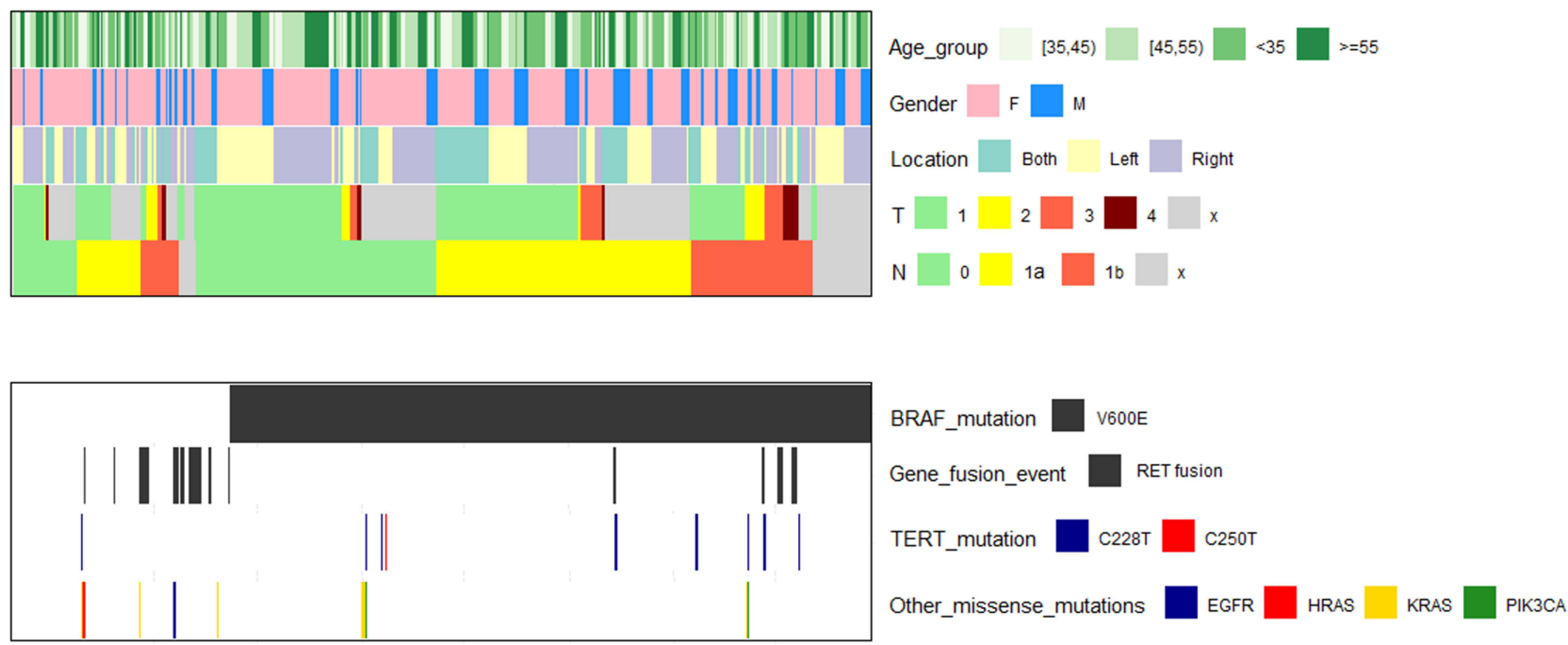

Figure I Clinical and genetic profile of 395 Chinese patients with papillary thyroid carcinoma (PTC). Up: clinical profile, including age, gender, T stage, N stage, and location of tumor. Down: genetic profile.

the left side than on the right side ( $p=0.028$, one-sided hypothesis test). All patients in our study had M0 disease (Document S1). The clinical characteristics of all patients are shown in Figure 1 and listed in Table 1.

At least 336 patients $(85.07 \%)$ had at least one driver gene; 310 patients ( $78.48 \%$ of the enrolled population) had $B R A F$ mutations, which represented the majority genotype (310/336, or 92\%). All $310 B R A F$ mutations were $B R A F^{V 600 E}$ mutations. Other driver genes included $R A S(\mathrm{n}=8), T E R T(\mathrm{n}=9), P I K C 3 A(\mathrm{n}=2)$, and EGFR $(\mathrm{n}=1)$. RET fusions $(\mathrm{n}=28$, or 7.09\%) were also identified; these included CCDC6 $(\mathrm{n}=12), N C O A 4(\mathrm{n}=8), A C B D 5(\mathrm{n}=3)$, intergenic_JMJD1C_REEP3: RET_12 $(\mathrm{n}=2), C C D C 186(\mathrm{n}=1), A F A P 1 L 2 \_6(\mathrm{n}=1), C E L F 2(\mathrm{n}=1)$, and ERC1 $(\mathrm{n}=1)$. Among them, CELF2, intergenic_JMJD1C_REEP3:RET_12, and CCDC186 fusion events were first reported in patients with PTC. Two or more driver gene mutations were detected in 20 individuals, of which 19 had BRAF mutations (95.00\%), 9 had TERT mutations (45.00\%), and 7 had RET mutations (35.00\%). One patient had three mutations: BRAF V600E,$T E R T$, and a RET: $A C B D 5$ gene fusion. Table 1 shows the clinicopathologic characteristics in different genotypes.

\section{Younger Age Was Associated with LNM and LLNM}

We performed a cross-sectional analysis to consider the relationship between clinical characteristics (age, gender, and location) and $\mathrm{T}$ and $\mathrm{N}$ stages (Table S1). According to ATA and American Joint Committee on Cancer (AJCC) guidelines, ${ }^{13,15} 55$ years is crucial for determination of the AJCC stage. ${ }^{13}$ Thus, we divided our study population into two groups: $\geq 55$ years and $<55$ years; we also grouped patients as $<45$ years and $\geq 45$ years according to the median age of our study (45 years). Fisher's exact test found no significant difference between clinical characteristics and $\mathrm{T}$ stage. However, patient age younger than 55 years (vs $\geq 55$ years) was associated with a higher $\mathrm{N}$ stage $(50.8 \%$ vs $43.3 \%$, $p=0.009)$. This finding held true when the cut-point changed to 45 years $(65.1 \%$ vs $46.0 \%, p<0.001)$. When age was considered as a continuous variable, no significant inflection points were identified. To eliminate the influence of gender and location, multi-dimensional contingency analyses were performed, with $\mathrm{CMH}$ test and exact conditional test adapted. The association still held true at a cutoff of 55 years $(p=0.015)$ or 45 years $(p<0.001)$, indicating that LNM tended to occur in younger patients in China. We also rule out whether different genotypes (especially RET fusions) could influence this phenomenon, but the age association remained in different genotype populations $(p=0.005)$.

\section{Higher BRAF Allele Frequency Reflected Advanced T and N Stages}

The $B R A F$ mutation was the most frequent mutation in the study $(\mathrm{n}=310 / 395)$. $T$-Test confirmed that compared with the $B R A F$-negative/RET-negative population, $B R A F$-positive patients tended to have an advanced T stage. Because the $B R A F$ 
Table I Clinical Features in Different Genotypes

\begin{tabular}{|c|c|c|c|c|c|c|c|c|}
\hline & \multicolumn{2}{|c|}{ Overall } & \multicolumn{2}{|c|}{ BRAF Positive } & \multicolumn{2}{|c|}{ RET Positive } & \multicolumn{2}{|c|}{ BRAF \& RET Negative } \\
\hline & \multicolumn{2}{|c|}{395} & \multicolumn{2}{|c|}{310} & \multicolumn{2}{|c|}{28} & \multicolumn{2}{|c|}{65} \\
\hline & No. & $\%$ & No. & $\%$ & No. & $\%$ & No. & $\%$ \\
\hline Mean age, years & \multicolumn{2}{|c|}{$44.43^{*}$} & \multicolumn{2}{|c|}{$44.74(p=0.737)^{*}$} & \multicolumn{2}{|c|}{$36.25(p=0.001)^{*}$} & \multicolumn{2}{|c|}{$45.89(p=0.408)^{*}$} \\
\hline$<45$ & 188 & 48.7 & 142 & 47.0 & 22 & 78.6 & 29 & 45.3 \\
\hline$\geq 45$ & 198 & 51.3 & 160 & 53.0 & 6 & 21.4 & 35 & 54.7 \\
\hline$<55$ & 296 & 76.7 & 230 & 76.2 & 27 & 96.4 & 46 & 71.9 \\
\hline$\geq 55$ & 90 & 23.3 & 72 & 23.8 & I & 3.6 & 18 & 28.1 \\
\hline Gender & & & \multicolumn{2}{|c|}{$p=0.608 *$} & \multicolumn{2}{|c|}{$p=0.660^{*}$} & \multicolumn{2}{|c|}{$p=0.119 *$} \\
\hline Male & 99 & 25.1 & 83 & 26.8 & 6 & 21.4 & II & 16.9 \\
\hline Female & 296 & 74.9 & 227 & 73.2 & 22 & 78.6 & 54 & 83.1 \\
\hline \multicolumn{9}{|l|}{ Location } \\
\hline Bilateral & 96 & 24.3 & 74 & 23.9 & 8 & 28.6 & 17 & 26.1 \\
\hline Right side & 166 & 42.0 & 130 & 42.0 & 14 & 50 & 25 & 38.5 \\
\hline Left side & 133 & 33.7 & 106 & 34.1 & 6 & 21.4 & 23 & 35.4 \\
\hline T stage & & & \multicolumn{2}{|c|}{$p=0.776^{*}$} & \multicolumn{2}{|c|}{$p=0.00 I^{*}$} & \multicolumn{2}{|c|}{$p<0.00 I^{*}$} \\
\hline TI & 198 & 78.0 & 160 & 78.1 & 6 & 27.3 & 32 & 91.4 \\
\hline $\mathrm{T} 2$ & 20 & 7.9 & 14 & 6.8 & 4 & 18.2 & 3 & 8.6 \\
\hline T3 & 23 & 9.0 & 21 & 10.2 & 5 & 22.7 & 0 & 0 \\
\hline $\mathrm{T} 4$ & 13 & 5.1 & 10 & 4.9 & 7 & 31.8 & 0 & 0 \\
\hline \multicolumn{2}{|l|}{$N$ stage } & & \multicolumn{2}{|c|}{$p=0.953^{*}$} & \multicolumn{2}{|c|}{$p<0.001 *$} & \multicolumn{2}{|c|}{$p=0.127^{*}$} \\
\hline No & 178 & 45.1 & 139 & 44.8 & 3 & 10.7 & 36 & 55.4 \\
\hline $\mathrm{Nla}$ & 145 & 36.7 & 116 & 37.4 & 8 & 28.6 & 23 & 35.4 \\
\hline NIb & 72 & 18.2 & 55 & 17.8 & 17 & 60.7 & 6 & 9.2 \\
\hline
\end{tabular}

Note: *The $t$-test was performed to examine the mean age, proportion of women, proportion of T3 and T4 stage, and proportion of NI stage between Overall patients and each subgroups classified by genotypes.

mutation is highly relevant with PTC, we collected the MAF of BRAF in 297 patients and explored the association between the MAF and the $\mathrm{T}$ and $\mathrm{N}$ stages (Figure 2). The MAF varied from $0.20 \%$ to $41.78 \%$, with a mean MAF of $11.21 \%$ (mean per side: left, 12.47\%; right, 10.06\%) (Document S1 and Figure S2). Post hoc tests revealed that advanced $\mathrm{T}$ and $\mathrm{N}$ stages were associated with higher mean $B R A F$ frequency in the overall population $(p<0.001$ and $p=0.004$ for $\mathrm{T}$ and $\mathrm{N}$ stage, respectively), especially in left-sided PTC ( $p=0.001$ and 0.007 for T and N stage, respectively). Plotting the cumulative positive rate of high-risk clinicopathologic features that varied with $B R A F$ allele frequency (Figure $\mathrm{S} 3$ ) showed that the risk of advanced $\mathrm{T}$ and $\mathrm{N}$ stage increases linearly as the $B R A F$ allele frequency increases. No significant platform or inflection point was found in our analysis.

As a result, patients were grouped to three groups according to MAF by lower quartile $(2.54 \%)$ and upper quartile (16.93\%). MAFs $<2.54 \%$ were considered the low-risk group, and MAFs $>16.93 \%$ were considered the high-risk group. Patients with a MAF between $2.54 \%$ and $16.93 \%$ were considered at intermediate risk. We calculated the odds of $\mathrm{T}$ and $\mathrm{N}$ stages in each group and determined the odds ratio (OR) between different groups. Results are shown in Table 2. The risks of LNM, LLNM, and T3 + T4 stage were significantly higher in the high-risk group compared with low-risk group (OR=3.38, 3.46 , and 8.54 , respectively) and the intermediate-risk group ( $\mathrm{OR}=2.04,2.07$, and 4.07 , respectively). Therefore, higher $B R A F$ allele frequency may be associated with a high risk of clinicopathologic features in Chinese patients with PTC. 

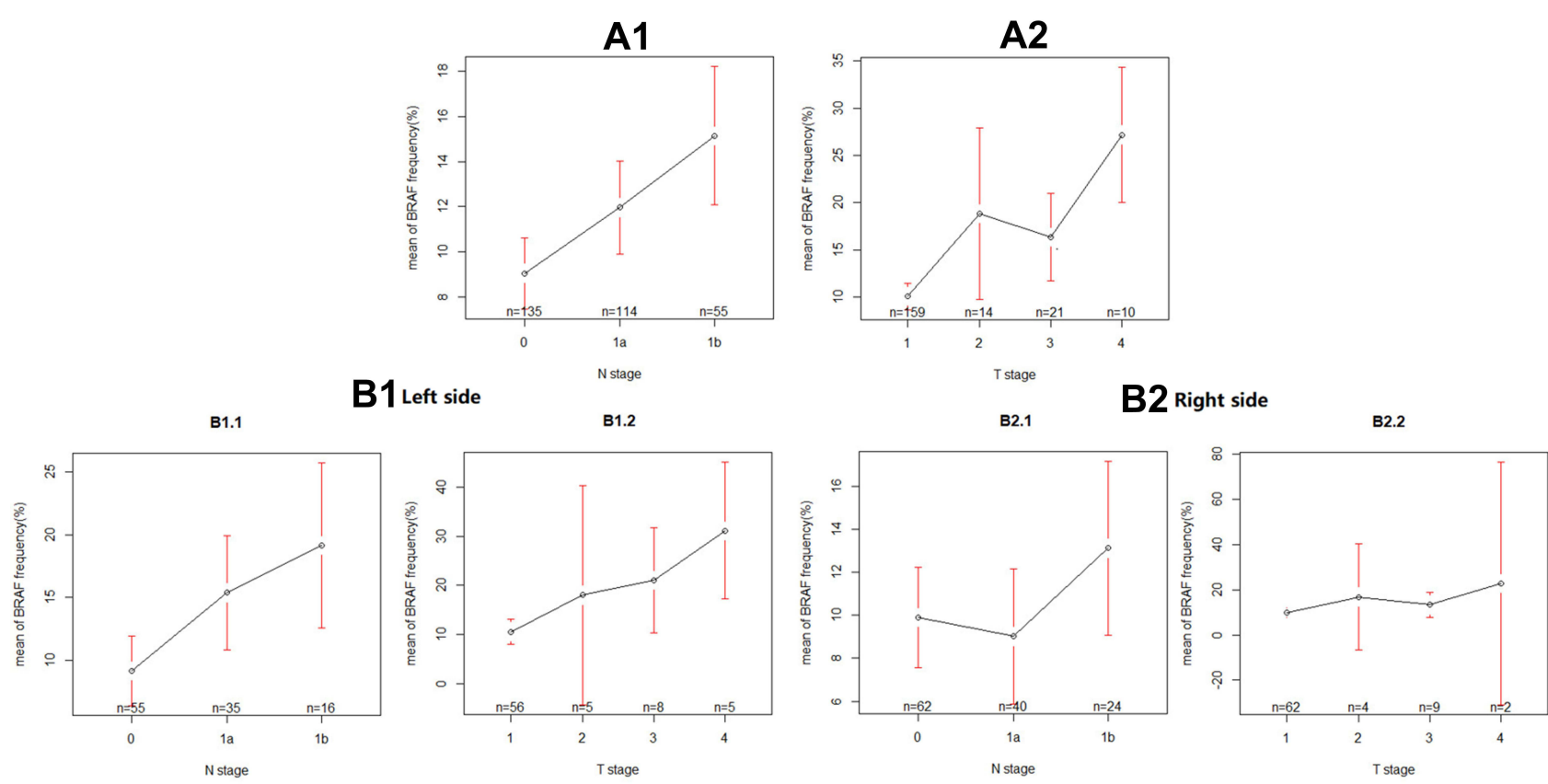

Figure 2 The relationship between mutation frequency at different primary foci locations and $\mathrm{T}$ and $\mathrm{N}$ staging in the BRAF-positive mutation population after correction for age and gender. (A) The relationship between gene frequency and $\mathrm{T}$ and $\mathrm{N}$ stages without considering location. (AI) $\mathrm{N}$ stage, (A2) T stage. (B) The relationship between gene frequency and $\mathrm{T}$ and $\mathrm{N}$ stage considering location. (BI) $\mathrm{N}$ stage, (BI.I) left side, (BI.2) right side, (B2) T stage, (B2.I) left side, (B2.2) right side.

\section{RET Fusions Significantly Indicated Advanced T and N Stages}

In our population, the $R E T$-positive patients $(\mathrm{n}=28)$ had quite distinctive clinical characteristics compared with the RETnegative population $(\mathrm{n}=367)$. They were younger, had larger tumor sizes, and had a significant propensity for LLNM (Table 1). OR calculations clarified the association between RET and the clinicopathologic features (ORs=7.60 for LNM, 8.77 for LLNM, and 10.40 for T3 + T4 compared with the RET-negative population) (Table 3). No significant effect of the location of the primary site on the clinical characteristics of the RET-positive population was found. Because many different genes are fused with RET, Fisher's exact test was performed to determine whether different fusions could lead to

Table 2 Odds Ratio Between Different Risk Groups in BRAF-Positive Patients

\begin{tabular}{|c|c|c|c|}
\hline Odds Ratio (NI vs N0) & Low Risk (Odds=0.727) & Intermediate Risk (Odds=I.202) & High Risk (Odds=2.454) \\
\hline High risk $(n=76)$ & 3.375 & \multirow[t]{3}{*}{2.041} & \\
\hline Middle risk $(n=152)$ & 1.654 & & \\
\hline \multicolumn{3}{|l|}{ Low risk $(n=76)$} & \\
\hline Odds Ratio (NIb) & Low Risk (Odds=0.1 I8) & Intermediate Risk (Odds=0.197) & High Risk (Odds=0.407) \\
\hline High risk ( $n=76)$ & 3.463 & \multirow[t]{2}{*}{2.070} & \\
\hline Middle risk $(n=152)$ & 1.673 & & \\
\hline \multicolumn{4}{|l|}{ Low risk $(\mathrm{n}=76)$} \\
\hline Odds Ratio (T3 + T4) & Low Risk (Odds=0.054) & Intermediate Risk (Odds=0.1 I3) & High Risk (Odds=0.462) \\
\hline High risk $(n=76)$ & 8.538 & \multirow[t]{3}{*}{4.070} & \\
\hline Intermediate risk $(n=\mid 52)$ & 2.098 & & \\
\hline Low risk $(\mathrm{n}=76)$ & & & \\
\hline
\end{tabular}


Table 3 Odds Ratio in Different Genotypes (A and B)

\begin{tabular}{|c|c|c|c|}
\hline Clinicopathological Stage & $p$ value* & Odds Ratio & Confidence Interval \\
\hline \multicolumn{4}{|c|}{ (A) RET-positive vs RET-negative disease } \\
\hline NI & 0.001 & 7.595 & {$[2.607,32.296]$} \\
\hline NIb & $<0.001$ & 8.767 & {$[3.943,20.266]$} \\
\hline $\mathrm{T} 3+\mathrm{T} 4$ & $<0.001$ & 10.40 & {$[4.081,27.217]$} \\
\hline \multicolumn{4}{|c|}{ (B) Multiple driver genes mutation vs single or no driver gene mutation } \\
\hline NI & 0.007 & 2.80 & {$[1.38,6.18]$} \\
\hline NIb & $<0.001$ & 7.04 & {$[3.56,14.07]$} \\
\hline $\mathrm{T} 3+\mathrm{T} 4$ & $<0.001$ & 7.48 & {$[3.31,16.98]$} \\
\hline
\end{tabular}

Note: *Examined by Fisher's exact test.

different clinicopathologic characteristics (Table S2). CCDC6, the major fusion partner with $R E T(\mathrm{n}=12 / 28)$, may be associated with a younger age of PTC onset and more LLNM $(p=0.12)$.

\section{Multiple Driver Gene Mutations and TERT Population}

In this study, $5.06 \%$ of the population $(\mathrm{n}=20 / 395)$ had at least two driver genes identified simultaneously, and nine patients had TERT mutations (Document S1). In a patient (case 444) with no BRAF mutation, HRAS and TERT mutations were identified. No $B R A F$-positive/RAS-positive patients were identified. Patients with at least two driver mutations were defined as the multiple driver gene mutated population. They tended to develop T3 or T4 stage disease more often, $(p<0.001, \mathrm{OR}=7.48, \mathrm{CI}=[3.31,16.98])$ and to have more $\mathrm{LNM}(p=0.007, \mathrm{OR}=2.80, \mathrm{CI}=[1.38,6.18])$ and LLNM $(p<0.001, \mathrm{OR}=7.04, \mathrm{CI}=[3.56,14.07])$ compared with patients who had single or no driver gene mutations (Table 4).

Interestingly, the TERT gene, a widely reported strong driver gene, was only found in the multi-mutant population and was frequently accompanied by almost simultaneous mutations in the $B R A F$ gene $(\mathrm{n}=8 / 9)$. The $T E R T$-positive population had an older mean age than the $B R A F$-positive population (55.56 years vs 44.74 years) but did not have larger tumor diameters or more LNM events (Table $\mathrm{S} 3$ ). These findings are consistent with previous reports of BRAF-positive/TERTpositive patients with PTC from Northeast China. ${ }^{14}$

\section{Novel RET Gene Fusions}

Three rare or novel RET fusion partners were identified in our study: CCDC186, CELF2, and intergenic region between $J M J D 1 C$ and REEP3. The 5' region of CCDC186 (exons 1-15) was connected to the 3' region of RET (exons 12-20), and the fusion was in frame. Using the emerging application of NGS, CCDC186:RET fusion has been newly noted in Chinese patients with lung cancer and thyroid cancer. In a cohort of Chinese patients with lung cancer, CCDC186 reportedly attached to RET at its intron $10 .{ }^{16}$ However, in another two cohorts of Chinese patients with PTC, CCDC186

Table 4 Clinical Characteristics of Multiple Driver Gene-Mutated Population

\begin{tabular}{|l|c|c|c|c|c|}
\hline Clinicopathologic Characteristics & Multiple Mutations & Overall & $\boldsymbol{p}$ value & No Mutation & $\boldsymbol{p}$ value* \\
\hline Mean age & 40.63 & 44.43 & 0.080 & 45.83 & 0.056 \\
Male & 0.268 & $0.25 \mathrm{I}$ & $0.8 \mathrm{II}$ & 0.136 & 0.115 \\
T3+ T4 & $0.44 \mathrm{I}$ & 0.142 & 0.002 & 0.000 & $<0.00 \mathrm{I}$ \\
NI & 0.756 & 0.549 & 0.006 & 0.458 & 0.002 \\
NIb & 0.688 & 0.288 & $<0.00 \mathrm{I}$ & 0.158 & $<0.00 \mathrm{I}$ \\
Bilateral & 0.268 & 0.243 & 0.732 & 0.254 & 0.877 \\
Right side (in unilateral) & 0.567 & 0.555 & 0.906 & 0.500 & 0.579 \\
\hline
\end{tabular}

Note: *The $t$-test was performed to examine the mean value and proportion of male, T3+T4, NI, NIb and locations. 
reportedly fused to RET at its intron $7 .^{12,17} C C D C 186$, as shown in its name, harbors coiled-coil domains, which mediate dimerization and the consequent activation of the kinase domain of RET.

$C E L F 2$, as a member of the $C E L F$ family, plays an important role in RNA co-transcription and post-transcriptional modifications. In a previous study, $C E L F 2$ inhibited the progression of non-small cell lung cancer by suppressing the binding of PTEN to PREX2. ${ }^{18}$ In addition, inactivation of CELF2 has been associated with gastric, ovarian, breast, and colorectal cancers. ${ }^{19-22}$ The $5^{\prime}$ region of $C E L F 2$ (exons 1-9) has attacked to exons 10-20 of RET, and the fusion was in frame.

A novel intergenic rearrangement was also identified in this cohort. RET was fused to a point $14.7 \mathrm{~kb}$ upstream of the coding region of the JMJDIC gene. The protein encoded by this gene interacts with thyroid hormone receptors and is thought to be a coactivator for key transcription factors. Because RNA was not available, the fusion was not confirmed at the RNA level. Several intergenic rearrangements, mostly to exon 12 of RET, have also been identified. ${ }^{23}$ Intergenic rearrangements accounted for approximately $7.7 \%$ of identified $R E T$ fusions. ${ }^{23}$ However, none of these intergenic $R E T$ rearrangements have responded to RET-related tyrosine kinase inhibitors. The significance of these intergenic rearrangements, including whether functional fusion RNAs can be transcribed from these intergenic rearrangements, remains to be determined.

\section{Discussion}

To our knowledge, this is the first multicenter study to examine the genetic characteristics of PTC and explore the relationships between the genotype and $\mathrm{T}$ and $\mathrm{N}$ disease stages in China. In this study, we defined high-risk clinicopathologic features as the presence of lymph node metastases (LNM, N1), the presence of lateral lymph node metastases (LLNM, N1b), and a large tumor diameter or invasion of surrounding tissue (T3 or T4). Here, we confirmed that high MAF of $B R A F, R E T$ fusions, and multiple driver gene mutations were related to those high-risk clinicopathologic features. Genetic monitoring of preoperative puncture biopsy tissue has been shown to assist in the diagnosis of benign or malignant tumors, therefore this study may help doctors assess the risk of LNM and thus guide the choice of surgical approach.

In the study population, $78.5 \%$ of the patients $(\mathrm{n}=310 / 395)$ had a $B R A F$ mutation, and all were the $B R A F^{V 600 E}$ mutation. Compared against data from The Cancer Genome Atlas ${ }^{24}$ (Table 5), the prevalence of BRAF mutation in this study was significantly higher than in a US PTC population $(59.7 \%, p<0.001)$, and $R A S(\mathrm{n}=8), T E R T(\mathrm{n}=9)$, and PIKC3A $(\mathrm{n}=2)$ were significantly less common. In contrast, the distribution of genotypes in this study was very similar to that of a previous single-centered study in China ${ }^{12}$ (in which $B R A F$ represented $72.4 \%$ of the patients), suggesting that the genetic profile of PTC may vary in different regions of the globe. Table S4 summarizes the genotype distribution of mutations in several regions and shows that the $B R A F$ mutation represented in all patients is predominant in Korea $(72 \%)^{25}$ but not in Japan $(38 \%)^{26,27}$ or Italy $(38.1 \%) .{ }^{28}$ Only $59.5 \%$ of the patients in Saudi Arabia carry a $B R A F$ mutation. ${ }^{29}$ We conclude that $B R A F$ is the main driver in this Chinese PTC population.

Table 5 Difference of Clinical and Genetic Profiles Compare Against TCGA Data

\begin{tabular}{|l|c|c|c|}
\hline & Chinese & TCGA & p value* \\
\hline Mean age & 44.43 & 46.79 & 0.013 \\
Male (\%) & $0.25 I$ & 0.268 & 0.570 \\
T3 + T4 (\%) & 0.142 & 0.347 & $<0.001$ \\
NI (\%) & 0.549 & 0.443 & 0.002 \\
NIb (\%) & 0.182 & 0.141 & 0.106 \\
Gene fusion events (\%) & 0.071 & 0.149 & $<0.001$ \\
RAS mutation (\%) & 0.020 & 0.105 & $<0.001$ \\
BRAF mutation (\%) & 0.785 & 0.508 & $<0.001$ \\
TERT mutation (\%) & 0.023 & 0.073 & $<0.00 \mathrm{I}$ \\
\hline
\end{tabular}

Note: *The $t$-test was performed to examine the mean value between data from this study and TCGA data. Abbreviation: TCGA, The Cancer Genome Atlas. 
Few studies have reported the influence of the MAF of $B R A F$ mutations in clinical behavior of PTC. Two previous studies (Guerra et $\mathrm{al}^{30}$ and Min-Hee $\mathrm{Kim}^{31}$ ) have reported that higher MAF of BRAF mutations is related to bigger tumor size and LNM in Italian and Korean people, which conflicted with Gandofi's report. ${ }^{32}$ In view of the fact that the genotype distribution (especially BRAF and RAS) in PTC is different in different races, further studies are needed in different races. We certified the influence of MAF of BRAF mutations in Chinese PTC population. As a result, the mean MAF of $B R A F$ mutation was higher in patients with advanced $\mathrm{T}$ and $\mathrm{N}$ stages (Figure 2), and the risk of advanced $\mathrm{T}$ and $\mathrm{N}$ stages increased linearly as the MAF increased (Figure S2). Besides, we firstly reported that increased MAF of BRAF is related to LLNM. Those conclusions strongly supported Guerra's finding, which may guide treatment and prognosis.

It has been proved that intratumoral heterogeneity (ITH) plays an important role in genesis and development of cancer, but it is debated in early PTC. Some studies discovered that ITH existed in different area of PTC samples, ${ }^{33}$ Masoodi et $\mathrm{al}^{34}$ studied 79 PTC samples and described the evolutionary patterns to prove existence of ITH in PTC. Fugazzola et $\mathrm{al}^{35}$ summarized recent studies about ITH in PTC and found that ITH may be not marginal event in early PTC. As a result, the varied MAF of $B R A F(0.20-41.78 \%)$ in our study may could be explained as ITH. But as a clinical study, we could not give a certain answer about this problem without further experiment. However, considering that most patients will not perform any test for ITH or purity of non-tumoral cells during gene detection, we believe that the conclusion that higher MAF of $B R A F$ is related with advanced stage may still hold true in clinical practice.

$R E T$ fusions have been widely reported as an indicator of poor prognosis in PTC. ${ }^{36,37}$ In this study, RET fusions were associated with younger age at onset (mean, 37 years), $\mathrm{LNM}(p=0.001, \mathrm{OR}=7.60, \mathrm{CI}=[2.61,32.30]), \mathrm{LLNM}(p<0.001$, $\mathrm{OR}=8.77, \mathrm{CI}=[3.94,20.27])$, and $\mathrm{T} 3+\mathrm{T} 4(p<0.001, \mathrm{OR}=10.40, \mathrm{CI}=[4.08,27.22])$, which coincide with Liang's study. ${ }^{12}$ Additional analysis revealed that CCDC6 fusion may be associated with LLNM $(p=0.12)$. However, previous research showed that, in US and European populations, PTC with a RET:CCDC6 gene fusion was usually inert and tended to hyper-differentiate. ${ }^{38}$ In a recent study, Liu et $\mathrm{al}^{17}$ noted that no significant difference was found in the Chinese population with RET:CCDC6 compared with a RET:non-CCDC6 population. RET:CCDC6 fusion events are very common in RET fusions, but more studies are needed to confirm the role of CCDC6 in PTC.

We also found that younger patients ( $<45$ years or $<55$ years) were more likely to have LNM. This conclusion still held true $(p=0.005)$ after a multi-dimensional contingency analysis was performed to control for the influence of genotype, notably RET fusions. According to AJCC guidelines, ${ }^{15}$ the AJCC stage of young patients ( $<55$ years) with $\mathrm{N} 1$ or N1b stage is less advanced than the stage in the older ( $>55$ years) population. However, this Chinese study revealed that the $\mathrm{N}$ stage in young patients was usually higher than that in older patients. Zhang et $\mathrm{al}^{39}$ also found that the Chinese patients with PTC who were $<40$ years old had greater tendencies to develop LNM $(10.7 \%$ vs $3.4 \%$, $p=0.006$ ). This finding suggests that more aggressive surgical approaches, such as prophylactic bilateral central lymph node dissection and prophylactic lateral lymph node dissection, should be applied for young patients with PTC in China.

There are several limitations to our study. First, this study was retrospective, so the integrity of data could not guaranteed. Second, selection bias may exist, because not every patient diagnosed with PTC was willing to participate in our study. Third, we used a DNA detection technique rather than RNA detection, and the detection mainly included 57 major high-frequency mutations; therefore, some mutations may not have been detected. For example, all gene fusion events in our dataset were associated with RET. Previously reported gene fusion events, such as NTRK, THADA, and $B R A F$, were not observed. Last, the cross-sectional design precluded exploration of factors associated with prognosis, and the lateral metastases or tumor diameter may not actually be associated with patient prognosis. Prospective study data may be needed to overcome these limitations.

In conclusion, our study reported the clinicopathologic characteristics and somatic gene mutation profile of PTC in a Chinese population, confirming that the main driver mutation is the BRAF mutation. This finding was significantly different from results in other countries or regions, therefore the biologic behavior of PTC may also differ significantly. We also confirmed some other conclusions. A high MAF of BRAF suggested a high risk of clinicopathologic characteristics. Tumors with RET fusion events or multiple driver gene mutations tended to display more malignant biologic behavior than tumors without these changes. Moreover, more LNM developed in young versus older patients in this Chinese population. Our study may help clinicians develop individualized treatment strategies according to genetic 
profiles. We will continue to observe these patients and investigate the relationship between genetic characteristics and risk of recurrence in the future.

\section{Ethics Approval Statement}

The study protocol was approved by the ethical board of the Chinese Academy of Medical Sciences and Peking Union Medical College Hospital, and all participants gave written informed consent at their local hospitals.

\section{Acknowledgments}

We thank the USCI Medical Laboratory Co, Ltd, for providing us with the genetic test results, data, and related assistance.

\section{Author Contributions}

All authors contributed significantly to the work reported, whether that is in the conception, study design, execution, acquisition of data, analysis and interpretation, or in all these areas; took part in drafting, revising or critically reviewing the article; gave final approval of the version to be published; have agreed on the journal to which the article has been submitted; and agree to be accountable for all aspects of the work.

\section{Funding}

This work was supported by the Personnel Training Program of the Third Military Medical University (No. XZ-2019505-045). The money was used to collect data from our patients.

\section{Disclosure}

The authors report no conflicts of interest in this work.

\section{References}

1. Li R, Wang Y, Du L. A rapidly increasing trend of thyroid cancer incidence in selected East Asian countries: joinpoint regression and age-periodcohort analyses. Gland Surg. 2020;9(4):968-984.

2. Yang L, Zheng RS, Wang N, et al. [Analysis of Incidence and Mortality of Thyroid Cancer in China, 2013]. Zhonghua Zhong Liu Za Zhi. 2017;39 (11):862-867. Chinese.

3. Du L, Li R, Ge M, et al. Incidence and mortality of thyroid cancer in China, 2008-2012. Chin J Cancer Res. 2019;31(1):144-151.

4. Sui C, Liang N, Du R, et al. Time trend analysis of thyroid cancer surgery in China: single institutional database analysis of 15,000 patients. Endocrine. 2020;68(3):617-628.

5. McLeod DS, Sawka AM, Cooper DS. Controversies in primary treatment of low-risk papillary thyroid cancer. Lancet. 2013;381(9871):1046-1057.

6. Ji YB, Yoo HS, Song CM, Park CW, Lee CB, Tae K. Predictive factors and pattern of central lymph node metastasis in unilateral papillary thyroid carcinoma. \#N/A. 2016;43(1):79-83.

7. Koo BS, Choi EC, Yoon YH, Kim DH, Kim EH, Lim YC. Predictive factors for ipsilateral or contralateral central lymph node metastasis in unilateral papillary thyroid carcinoma. Ann Surg. 2009;249(5):840-844.

8. Ge J, Wang J, Wang H, et al. The BRAF V600E mutation is a predictor of the effect of radioiodine therapy in papillary thyroid cancer. $J$ Cancer. 2020;11(4):932-939.

9. Xing M. Molecular pathogenesis and mechanisms of thyroid cancer. \#N/A. 2013;13(3):184-199.

10. Liu R, Bishop J, Zhu G, Zhang T, Ladenson PW, Xing M. Mortality risk stratification by combining BRAF V600E and TERT promoter mutations in papillary thyroid cancer: genetic duet of BRAF and TERT promoter mutations in thyroid cancer mortality. JAMA Oncol. 2017;3 (2):202-208.

11. Li C, Lee KC, Schneider EB, Zeiger MA. BRAF V600E mutation and its association with clinicopathological features of papillary thyroid cancer: a meta-analysis. J Clin Endocrinol Metab. 2012;97(12):4559-4570.

12. Liang J, Cai W, Feng D, et al. Genetic landscape of papillary thyroid carcinoma in the Chinese population. J Pathol. 2018;244(2):215-226.

13. Haugen BR, Alexander EK, Bible KC, et al. 2015 American Thyroid Association Management Guidelines for Adult Patients with Thyroid Nodules and Differentiated Thyroid Cancer: the American Thyroid Association Guidelines Task Force on Thyroid Nodules and Differentiated Thyroid Cancer. Thyroid. 2016;26(1):1-133.

14. Tsuchiya A, Koizumi M, Ohtani H. World Health Organization Classification (2004)-based re-evaluation of 95 nonfunctioning "malignant" pancreatic endocrine tumors reported in Japan. Surg Today. 2009;39(6):500-509.

15. Tuttle RM, Haugen B, Perrier ND. Updated American Joint Committee on Cancer/Tumor-Node-Metastasis Staging System for Differentiated and Anaplastic Thyroid Cancer (Eighth Edition): what changed and why? Thyroid. 2017;27(6):751-756.

16. Zhang K, Chen H, Wang Y, et al. Clinical characteristics and molecular patterns of RET-Rearranged lung cancer in Chinese patients. Oncol Res. 2019;27(5):575-582. 
17. Liu Y, Wu S, Zhou L, Guo Y, Zeng X. Pitfalls in RET fusion detection using break-apart FISH probes in papillary thyroid carcinoma. $J$ Clin Endocrinol Metab. 2020;106(4):1129.

18. Yeung YT, Fan S, Lu B, et al. CELF2 suppresses non-small cell lung carcinoma growth by inhibiting the PREX2-PTEN interaction. Carcinogenesis. 2020;41(3):377-389.

19. Ramalingam S, Ramamoorthy P, Subramaniam D, Anant S. Reduced expression of RNA binding protein CELF2, a putative tumor suppressor gene in colon cancer. Immunogastroenterology. 2012;1(1):27-33.

20. Guo Q, Wu Y, Guo X, et al. The RNA-binding protein CELF2 inhibits ovarian cancer progression by stabilizing FAM198B. Mol Ther Nucleic Acids. 2021;23:169-184.

21. Pique L, Martinez de Paz A, Pineyro Det al. Epigenetic inactivation of the splicing RNA-binding protein CELF2 in human breast cancer. Oncogene. 2019;38(45):7106-7112.

22. Wang J, Liu L, Sun Y, et al. miR-615-3p promotes proliferation and migration and inhibits apoptosis through its potential target CELF2 in gastric cancer. Biomed Pharmacother. 2018;101:406-413.

23. Ou S-HI, Zhu VW. Catalog of 5' fusion partners in RET+ NSCLC Circa 2020. JTO Clinical and Res Rep. 2020;1(2):100037.

24. Cancer Genome Atlas Research N. Integrated genomic characterization of papillary thyroid carcinoma. Cell. 2014;159(3):676-690.

25. Hong AR, Lim JA, Kim TH, et al. The frequency and clinical implications of the BRAF(V600E) mutation in papillary thyroid cancer patients in Korea over the past two decades. Endocrinol Metab (Seoul). 2014;29(4):505-513.

26. Nasirden A, Saito T, Fukumura Y, et al. In Japanese patients with papillary thyroid carcinoma, TERT promoter mutation is associated with poor prognosis, in contrast to BRAF (V600E) mutation. Virchows Archiv. 2016;469(6):687-696.

27. Ito Y, Yoshida H, Maruo R, et al. BRAF mutation in papillary thyroid carcinoma in a Japanese population: its lack of correlation with high-risk clinicopathological features and disease-free survival of patients. Endocr J. 2009;56(1):89-97.

28. Fugazzola L, Puxeddu E, Avenia N, et al. Correlation between B-RAFV600E mutation and clinico-pathologic parameters in papillary thyroid carcinoma: data from a multicentric Italian study and review of the literature. Endocr Relat Cancer. 2006;13(2):455-464.

29. Rashid FA, Munkhdelger J, Fukuoka J, Bychkov A. Prevalence of BRAF(V600E) mutation in Asian series of papillary thyroid carcinoma-a contemporary systematic review. Gland Surg. 2020;9(5):1878-1900.

30. Guerra A, Fugazzola L, Marotta V, et al. A high percentage of BRAFV600E alleles in papillary thyroid carcinoma predicts a poorer outcome. $J$ Clin Endocrinol Metab. 2012;97(7):2333-2340.

31. Kim MH, Bae JS, Lim DJ, et al. Quantification of BRAF V600E alleles predicts papillary thyroid cancer progression. Endocr Relat Cancer. 2014;21(6):891-902.

32. Gandolfi G, Sancisi V, Torricelli F, et al. Allele percentage of the BRAF V600E mutation in papillary thyroid carcinomas and corresponding lymph node metastases: no evidence for a role in tumor progression. J Clin Endocrinol Metab. 2013;98(5):E934-942.

33. Guerra A, Sapio MR, Marotta V, et al. The primary occurrence of BRAF(V600E) is a rare clonal event in papillary thyroid carcinoma. $J$ Clin Endocrinol Metab. 2012;97(2):517-524.

34. Masoodi T, Siraj AK, Siraj S, et al. Evolution and impact of subclonal mutations in papillary thyroid cancer. Am J Hum Genet. 2019;105 (5):959-973.

35. Fugazzola L, Muzza M, Pogliaghi G, Vitale M. Intratumoral genetic heterogeneity in papillary thyroid cancer: occurrence and clinical significance. Cancers (Basel). 2020;12:2.

36. Sugg SL, Ezzat S, Zheng L, Freeman JL, Rosen IB, Asa SL. Oncogene profile of papillary thyroid carcinoma. Surgery. 1999;125(1):46-52.

37. Musholt TJ, Staubitz JI, Antonio Camara RJ, et al. Detection of RET rearrangements in papillary thyroid carcinoma using RT-PCR and FISH techniques - a molecular and clinical analysis. Eur J Surg Oncol. 2019;45(6):1018-1024.

38. Acquaviva G, Visani M, Repaci A, et al. Molecular pathology of thyroid tumours of follicular cells: a review of genetic alterations and their clinicopathological relevance. Histopathology. 2018;72(1):6-31.

39. Hu D, Zhou J, He W, et al. Risk factors of lateral lymph node metastasis in cN0 papillary thyroid carcinoma. World J Surg Oncol. 2018;16(1):30.

OncoTargets and Therapy

Dovepress

\section{Publish your work in this journal}

OncoTargets and Therapy is an international, peer-reviewed, open access journal focusing on the pathological basis of all cancers, potential targets for therapy and treatment protocols employed to improve the management of cancer patients. The journal also focuses on the impact of management programs and new therapeutic agents and protocols on patient perspectives such as quality of life, adherence and satisfaction. The manuscript management system is completely online and includes a very quick and fair peer-review system, which is all easy to use. Visit http://www.dovepress.com/testimonials.php to read real quotes from published authors.

Submit your manuscript here: https://www.dovepress.com/oncotargets-and-therapy-journal 